\title{
Programa de educação pelo trabalho para a saúde: um relato de experiência
}

\author{
Educational program through work for health: an experience report
}

\author{
Jeferson Santos Araujo ${ }^{1}$, Glenda Marreira Vidal ${ }^{2}$, Felipe Nunes Brito ${ }^{3}$, Clisse Michele \\ Rodrigues Alves ${ }^{3}$, Djeane Kathe Mascote Leite ${ }^{4}$, Claudia Daniele \\ Tavares Dutra ${ }^{5}$, Carla Andréa Avelar Pires ${ }^{6}$
}

\begin{abstract}
RESUMO
Objetivo: O presente estudo tem como objetivo descrever a experiência vivenciada por acadêmicos a cerca do trabalho em equipe frente à proposta do programa de educação pelo trabalho para a saúde.

Descrição da experiência: Os discentes tiveram a oportunidade de vivenciarem, antes mesmo da sua formação, a experiência de funcionamento das equipes multiprofissionais no que tange à realidade do quadro da estratégia saúde da família, implementando ações assistenciais na comunidade e fortalecendo o tripé ensino, pesquisa e extensão.

Conclusão: A experiência de trabalho em equipe do programa ainda é incipiente, porém apresenta-se como um modelo assistencial a ser reproduzido pelos gestores, permitindo aos estudantes expandirem seus conhecimentos com a inserção precoce nos campos práticos e com a vivência de novas realidades e serviços.
\end{abstract}

Palavras-chave: saúde pública; ensino; saúde.

\section{ABSTRACT}

Objective: This study aims to describe an experience for students about teamwork in relation to the proposed program of education through work for health.

Description of experience: The students had the opportunity to experience, even before their formation, the operating process of multidisciplinary teams in relation to the context of the family health strategy reality, implementing community care actions and strengthening the tripod teaching, research and extension.

Conclusion: The teamwork experience in the program is still incipient, but presents itself as a model of care to be reproduced by managers, allowing students to expand their knowledge with early integration in the fields and with the practical experience of new realities and services.

Keywords: public health; teaching; health.

\footnotetext{
${ }^{1}$ Enfermeiro. Ex-Bolsista PET. Especialista em Enfermagem do Trabalho (UNINTER). Mestrando em Enfermagem Fundamental (USP). Vinculado ao Núcleo de Grupo de Estudo da Reabilitação de Pacientes Cirúrgicos e Oncológicos (USP).

${ }^{2}$ Nutricionista. Ex-Bolsista PET. Mestranda do Programa de Pós-Graduação em Nutrição (UFSC). Membro do Núcleo de Pesquisa de Nutrição em Produção de Refeições.

${ }^{3}$ Acadêmico de Medicina pela UFPA. Bolsista do PET-Saúde Ananindeua.

${ }^{4}$ Enfermeira. Preceptora do PET-Saúde Ananindeua.

${ }^{5}$ Nutricionista. Mestre em Doenças Tropicais. Docente da Faculdade de Nutrição (UFPA). Tutora do PET-Saúde Ananindeua.

${ }^{6}$ Médica. Mestre em Doenças Tropicais. Docente da Faculdade de Medicina (UFPA). Tutora do PET-Saúde Ananindeua.
} 


\section{INTRODUÇÃO}

A atenção básica dispersada pelas equipes de saúde preconizadas pelo Ministério da Saúde caracteriza-se por um conjunto de ações voltadas ao âmbito individual e coletivo que abrangem os níveis assistenciais da promoção, proteção e prevenção da saúde, através da realização de diagnósticos, tratamentos e a manutenção da saúde.

No Brasil, o termo caracteriza o primeiro nível de atenção à saúde, o que se apresenta teoricamente, como o contato inicial do paciente com os serviços ofertados pelo Sistema Único de Saúde (SUS). Seguindo esses preceitos, neste nível é desvelado como foco prioritário o desenvolvimento do programa governamental de assistência e desenvolvimento da Estratégia Saúde da Família (ESF) ${ }^{1}$.

A ESF foi implementada na década de 90 que, no contexto da política de saúde brasileira, deveria contribuir para a construção e consolidação do SUS. Embasada nas diretrizes do SUS, a ESF traz no centro de sua proposta a expectativa relativa à reorientação do modelo assistencial a partir da atenção básica ${ }^{2}$. Os ganhos e os limites superados a partir desta proposta têm alimentado discussões, cujos argumentos expõem a reflexão crítica sobre as práticas de saúde desenvolvidas no contexto das unidades de saúde da família.

Nesse contexto, a ESF auxilia na otimização e execução dos preceitos preconizados para a atenção básica, uma vez que, possibilita traçar um diagnóstico da situação de saúde da população assistida pela equipe, que servem como aporte para o planejamento preciso de atividades orientadas e focadas, segundo critérios de agravo à saúde, priorizando solução dos problemas em articulação continua e permanente com os indivíduos, famílias e comunidades assistidas pelo programa $^{3}$.

Os programas de ESF devem ser compostos por uma equipe multiprofissional, composta no mínimo por um profissional médico, enfermeiro, cirurgião dentista, auxiliar de consultório dentário, técnico de enfermagem e agente comunitário de saúde ${ }^{3,4}$, sendo uma das características do programa a preconização do trabalho em equipe a fim de garantir aos usuários uma assistência multiprofissional humanizada e individualizada que convertam atributos de cuidados indispensáveis para a preservação e promoção da saúde ${ }^{5}$.

O trabalho multiprofissional dentro da ESF funciona como um laço desafiador para o profissional, uma vez que, requer dos trabalhadores a superação dos limites de suas especialidades, exigindo que os mesmos desenvolvam um comportamento compartilhado de informação e implementações assistenciais voltadas ao indivíduo como um todo, não se restringindo, apenas, a sua "sintomatização" aparente do abalamento do seu processo saúde/doença, sendo voltado a todas as características definidoras da saúde do individuo, dessa forma necessitando da participação conjunta dos profissionais para a implementação de um tratamento ${ }^{4,6}$. A participação na equipe multiprofissional de saúde é uma prática incentivada pelas Diretrizes Curriculares Nacionais que norteiam as bases curriculares dos cursos da saúde que devem ser implementadas, repassadas e fortalecidas entre os acadêmicos da área da saúde, ainda dentro da academia, entrelaçando, assim, saberes e práticas comunitárias que vão de encontro ao auxílio de outras áreas da saúde a fim de promover um olhar mais holístico para os desafios encontrados na prática da saúde pública, consagrando o território de atuação de cada profissional da saúde e detectando os pontos primordiais para a práxis assistencial $^{7}$.

Dessa forma, o Programa de Educação pelo Trabalho para a Saúde (PET - Saúde) criado pela cooperação conjunta entre os Ministérios da Saúde e da Educação, apresenta-se aos centros de formação de recursos humanos da saúde como uma ferramenta destinada a promover maior aproximação e interação dos grupos de discentes de várias áreas com a comunidade assistida pela ESF, viabilizando programas de aperfeiçoamento e especialização em serviço dos profissionais da saúde, caracterizando-se como uma estratégia pedagógica que potencializa uma formação generalista/holística com práticas formativas contextualizadas e reflexivas sobre 0 trabalho em equipe focado na assistência comunitária ${ }^{8}$.

O programa PET - Saúde foi regulamentado pela portaria interministerial no 421, de 03 de março de 2010 
e inspirado no Programa de Educação Tutorial (PET) já existente no Ministério da Educação. Dessa forma a proposta do PET - Saúde implementada no município de Ananindeua - PA, vinculado a ESF - Seringal encontrase de acordo com a formação proposta pelas Diretrizes Curriculares dos Ministérios da Educação e da Saúde, de inserir os acadêmicos da área da saúde na interdisciplinaridade e no contexto da saúde pública, a fim de que vivenciem antes mesmos da sua formação, a experiência de funcionamento das equipes interdisciplinares e multiprofissionais no que tange à realidade da saúde pública.

De acordo com o exposto, o presente estudo objetiva descrever a experiência de acadêmicos inseridos no PET - Saúde Ananindeua a cerca do trabalho em equipe prestado à comunidade Seringal no ano de 2011.

\section{DESCRIÇÃO DA EXPERIÊNCIA}

A Proposta do grupo PET - Saúde desenvolvida no município de Ananindeua segue um cronograma previamente elaborado para o período de 24 meses, a qual busca novas metodologias e procedimentos que podem ser alcançadas e realizadas através da interação entre ensino, pesquisa, extensão e serviço com a realidade da comunidade onde estar inserida. Por meio de levantamentos de dados extraídos diretamente dos pacientes que frequentam a unidade da ESF - Seringal e das fichas ambulatoriais individuais de cada paciente cadastrado. A equipe identifica os agravos de saúde da população e elabora programas específicos que possam ser implementados, planejados e executados com eficiência pela comunidade, indo de encontro aos preceitos de promoção e prevenção da saúde coletiva.

O projeto vigora, desde abril de 2010, com a participação de doze equipes compostas por alunos dos cursos de graduação em medicina, nutrição, enfermagem e odontologia da Universidade Federal do Pará, com a participação efetiva de doze preceptores e dois tutores. Os discentes são acompanhados por preceptores, membros da equipe do Programa Saúde da Família da comunidade, e tutores docentes membros do programa.

A ESF está presente no município de Ananindeua desde 1998 e conta atualmente com 83 equipes de saúde da família, distribuídas em 5 (cinco) Pólos Sanitários assim distribuídos: Pólo I BR-Sul; Pólo II Distrito Industrial; Pólo III PAAR; Pólo IV Cidade Nova, Icuí Guajará e Icuí Laranjeira e Pólo V Jaderlândia. A ESF - Seringal está localizada no bairro do coqueiro, cidade nova VIII, Pólo IV e funciona no município desde dezembro de 2007

A área de atuação da ESF - Seringal é dividida em 07 (sete) microáreas. Tendo 1.071 famílias cadastradas e 4.312 pessoas sob a responsabilidade de uma equipe composta por 01 (uma) Médica, 01 (um) Odontólogo, 01 (uma) Enfermeira, 07 (sete) Agentes Comunitários de Saúde (ACS) e 01 (uma) Técnica de Enfermagem.

Os atendimentos prestados a população adstrita obedecem às normas do programa PET - Saúde e são realizados através do acompanhamento de visitas domiciliares, as quais permitem aferir em locos a realidade da população assistida, e permite o desenvolvimento de atividades como: auxílio no agendamento de consultas, atendimentos de eventuais procedimentos e atividades de educação à saúde.

A presença do programa na comunidade contribuiu para aumentar as possibilidades de humanização dos serviços de saúde e a formação dos futuros profissionais, instigando, nos mesmos, o comprometimento com o bem estar da sociedade e a prática responsável de ações pela evolução do setor da saúde, uma vez que o contato prévio com as dificuldades e agravos enfrentados pelos os usuários da ESF proporcionam à equipe PET - Saúde a construção de espaços de debate e consolidação de saberes, os quais aprimoram a compreensão do processo saúde e doença da comunidade assistida.

Sem contemplar, simultaneamente, ou mesmo, inferir na articulação das ações e dos saberes existentes pela comunidade, o programa proporcionou aos discentes a realização de palestras e círculos de debates educativos com a comunidade que permitiu a troca de experiências, conceitos e práticas entre os sujeitos, o qual facilitou a comunidade ter acesso ao conhecimento de educação e saúde, sem mesmo sair de seus domicílios, incentivando assim, a promoção de saberes, que vão de encontro ao autocuidado dos indivíduos pertencentes ao grupo. 
Para os discentes, o programa funciona como um verdadeiro laboratório de saúde coletiva ao ar livre, ao qual se recebe conhecimentos, tanto dos professores, preceptores, tutores, quanto da comunidade, sendo essa última detentora do saber consensual legitimamente elaborado nas suas praticas cotidianas, alicerçados na cultura local onde estão inseridos que ditam as formas como os mesmos guiam suas ações de saúde.

Com o PET - Saúde, os estudantes tiveram a oportunidade de se inserir na iniciação à pesquisa científica com foco em temas atuais, discutindo metodologias e programas de atenção na saúde pública, como a atenção a saúde do idoso, da mulher, da criança e do adolescente, com a realização de pesquisas estruturadas no atendimento domiciliar e ambulatorial. Pois, as pesquisas de campo deram suporte às ações preventivas, visto que, a partir delas foi possível realizar um diagnóstico inicial das condições de saúde da comunidade estudada e elaborar um plano de trabalho voltado à realidade local.

Por meio do trabalho em equipe, se enfatizou o trabalho preventivo com a população local dividindo-se a experiência da realização de visitas domiciliares aos pacientes acamados, incentivando a vacinação das diversas faixas etárias do programa de vacinação preconizado pelo Ministério da Saúde, realizando palestras e atividades para diversos grupos de risco para doenças crônicas não transmissíveis, acompanhando o programa Hiper-dia, realizando orientação nutricional, acompanhando a saúde física psicológica e a adesão ao tratamento do paciente, o qual por meio destes, auxilia-se na promoção da melhoria das condições de saúde da população da ESF - Seringal.

A proposta de trabalho em equipe do PET - Saúde Ananindeua ainda é incipiente, porém já renderam aos participantes grandes conhecimentos e a comunidade, bons resultados. A multiprofissionalidade e o bom entrosamento da equipe possibilitam a execução de ações práticas e rápidas, tornando-se, assim, um modelo de qualificação profissional, onde tanto a comunidade, quanto os profissionais envolvidos no processo de formação e os alunos, estão sendo beneficiados na perspectiva da construção de um novo saber coletivo e participativo, o qual permite aos estudantes expandirem seus conhecimentos com a inserção precoce nos campos práticos e com a vivência de novas realidades e serviços.

\section{DISCUSSÃO}

A Proposta de reunir uma equipe composta por acadêmicos de vários cursos em um único programa tem sido veiculada como uma estratégia para enfrentar o intenso processo de especialização na área da saúde, que no cenário atual, vem expandindo-se cada vez mais. Esse processo tende a aprofundar verticalmente o conhecimento acadêmico e a intervenção prática dos mesmos em aspectos individualizados das necessidades de saúde, que neste caso, foram vivenciados pelos discentes por meio das visitas domiciliares e do acompanhamento ambulatorial que esteve constantemente presente na unidade básica de saúde.

O programa PET - Saúde contempla essa característica através de suas vertentes disciplinares, sendo desenvolvido por estudantes que realizam atividades nos serviços de saúde a fim de facilitar o processo de integração entre o ensino, os serviços e a comunidade, tendo como princípio estimular a inserção das necessidades dos serviços na produção de conhecimento nos cursos de graduação ${ }^{8}$.

A aproximação do aluno à Atenção Básica permite que o mesmo implemente seus conhecimentos técnicos científicos adquiridos no centro de formação ao campo de prática, impulsionando dessa maneira o desenvolvimento de um ensino de qualidade voltado para as ações da saúde pública, a qual prima por desenvolver serviços de saúde a comunidade, levando em conta este cenário como um campo de aprendizagem capaz de desenvolver, nos futuros profissionais, competências para a realização de atividades assistenciais, gerenciais, de ensino e pesquisa $^{9}$.

Esta aproximação previa constitui-se em um instrumento que viabiliza os programas de aperfeiçoamento e especialização em serviço dos aspirantes da saúde, bem como suas iniciações ao 
campo de trabalho, estágios e vivências, indo de encontro às necessidades de fortalecimento das diretrizes do SUS aspiradas para a melhoria da assistência $^{8-10}$.

A implementação do programa trouxe a possibilidade para que o Ministério da Saúde cumpra seu papel constitucional de ordenador da formação de profissionais de saúde por meio da indução e do apoio ao desenvolvimento dos processos formativos necessários em todo o país, de acordo com características sociais e regionais, estimulando, assim, a formação de profissionais e docentes de elevada qualificação técnica e científica, bem como a atuação profissional pautada no espírito crítico reflexivo multiprofissional sobre a saúde publica, pela cidadania e pela função social da educação superior, orientados pelo princípio da indissociabilidade entre ensino, pesquisa e extensão, preconizado pelo Ministério da Educação, desenvolvendo atividades acadêmicas de ensino e aprendizado, mediante grupos que estimulem o aprendizado de natureza coletiva e interdisciplinar ${ }^{11}$.

A experiência vivenciada contribui para 0 fortalecimento e implementação das Diretrizes Curriculares Nacionais dos cursos de graduação da área da saúde. Visto sobre este enfoque, estimulou a formação de profissionais de saúde com perfil adequado às necessidades e às políticas de saúde vigentes, fomentam a articulação entre ensino e serviço, induzindo o provimento e favorecendo para uma futura fixação de profissionais de saúde capazes de promover a qualificação e a articulação multiprofissional da atenção à saúde em todo o território nacional, sensibilizando e preparando os futuros profissionais para 0 adequado enfrentamento das diferentes realidades de vida e de saúde da população brasileira, atrelado as características de cada cenário ${ }^{7,9,11}$.

Entretanto, a estratégia PET - Saúde apesar das grandes contribuições e conquistas, ainda necessita ser aperfeiçoada, fortalecida e defendida a fim de multiplicar esta iniciativa a diversos centros formadores de recursos humanos na área da saúde distribuídos no país, possibilitando a elaboração de projetos multicêntricos e multiprofissionais, a inserção das instituições de ensino e saúde na rede de atenção básica, articulando estratégias entre ensino e serviço para a formação qualificada dos profissionais voltados aos serviços de saúde, projetados para o desenvolvimento da atenção básica, dentro da ESF, ancorados no tripé ensino, pesquisa e extensão das Universidades.

\section{REFERÊNCIAS}

1. BRASIL. Ministério da Saúde. Política nacional de atenção básica. Brasília, DF; 2006. Série Pactos pela Saúde.

2. Dias V, Silveira D, Witt R. Educação em saúde: protocolo para o trabalho de grupos em atenção primária. Rev APS. abr./jun. 2009; 12(2):221-7.

3. Sousa MF, Hamann EM. Programa Saúde da Família no Brasil: uma agenda incompleta. Rev Ciênc Saúde Colet. 2009;14:1325-35.

4. Loch-Neckel G, Seemann G, Eidt HB, Rabuske MM, Crepaldi MA. Desafios para a ação interdisciplinar na atenção básica: implicações relativas à composição das equipes de saúde da família. Rev Ciênc Saúde Colet. 2009; 14:1463-72.

5. Conill EM. Ensaio histórico-conceitual sobre a Atenção Primária à Saúde: desafios para a organização de serviços básicos e da Estratégia Saúde da Família em centros urbanos no Brasil. Rev Cad Saúde Pública. 2008; 24(1):7-16.

6. Berardinelli LMM, Santos MLC. Repensando a interdisciplinaridade e 0 ensino de enfermagem. Rev Texto \& Contexto Enferm. 2005; 14(3):419-26.

7. Alves VS. Um modelo de educação em saúde para o Programa Saúde da Família: pela integralidade da atenção e reorientação do modelo assistencial. Interface Comun Saúde Educ 2005; 9(16):39-52

8. Santos BRL, Thiesen FV. O programa de educação pelo trabalho para a saúde fortalecendo a interdisciplinaridade na formação dos profissionais da área da saúde. Rev Ciência \& Saúde. 2010; 3(1):1.

9. Nascimento MS, et al. Oficinas pedagógicas: construindo estratégias para a ação docente - relato de experiência. Rev Saúde Com. 2007; 3(1):85-95.

10. Soubhia Z, Garanhani ML, Dessunti EM. O significado de aprender a pesquisar durante a graduação. Rev Bras Enferm. 2007; 60(2):178-83.

11. Araújo D, Miranda MCG, Brasil SL. Formação de profissionais de saúde na perspectiva da integralidade. Rev Baiana de Saúde Pública. 2007; 31:20-31.

\section{Endereço para correspondência:}

Jeferson Santos Araujo

Rua Machado de Assis, 1034

Ribeirão Preto/SP - CEP 14050-490

Telefone: +551682339606

E-mail: jeferson-ma@ig.com.br 